\title{
Micromechanics as a Testbed for Artificial Intelligence Methods Evaluation
}

\author{
Ernst Kussul ${ }^{1}$, Tatiana Baidyk ${ }^{1}$, Felipe Lara-Rosano ${ }^{1}$, Oleksandir Makeyev ${ }^{2}$, \\ Anabel Martin ${ }^{\prime}$ and Donald Wunsch ${ }^{3}$ \\ 1 Center of Applied Sciences and Technological Development, National \\ Autonomous University of Mexico, Cd. Universitaria, Circuito Exterior s/n, \\ Coyoacán, 04510, México, D.F., Mexico \\ ekussul@servidor.unam.mx; tbaidyk@aleph.cinstrum.unam.mx; \\ lararf@servidor.unam.mx; anabelmartin@lycos.com \\ 2 Dept. of Electrical and Computer Engineering, Clarkson University, \\ 5720, Potsdam, NY 136992, USA \\ mckehev@hotmail.com \\ 3 Applied Computational Intelligence Lab, Dept. of Electrical and \\ Computer Engineering, University of Missouri-Rolla, 1870 Miner \\ Circle, Rolla MO 65409, USA \\ dwunsch@ece.umr.edu
}

\begin{abstract}
Some of the artificial intelligence (AI) methods could be used to improve the performance of automation systems in manufacturing processes. However, the application of these methods in the industry is not widespread because of the high cost of the experiments with the AI systems applied to the conventional manufacturing systems. To reduce the cost of such experiments, we have developed a special micromechanical equipment, similar to conventional mechanical equipment, but of a lot smaller overall sizes and therefore of lower cost. This equipment can be used for evaluation of different AI methods in an easy and inexpensive way. The methods that show good results can be transferred to the industry through appropriate scaling. This paper contains brief description of low cost microequipment prototypes and some $\mathrm{AI}$ methods that can be evaluated with mentioned prototypes.
\end{abstract}

\section{Introduction}

The development of AI technologies gives one an opportunity to use them not only for conventional applications (expert systems, intelligent databases [1], technical diagnostics $[2,3]$ etc.) but also for automation of mechanical manufacturing. Such AI methods as adaptive critic design $[4,5]$, adaptive fuzzy Petri networks $[6,7]$,

Please use the following format when citing this chapter:

Kussul, E., Baidyk, T., Lara-Rosano, F., Makeyev, O., Martin, A., Wunsch, D., 2006, in IFIP International Federation for Information Processing, Volume 218, Professional Practice in Artificial Intelligence, eds. J. Debenham, (Boston: Springer), pp. $275-284$ 
neural network based computer vision systems [8-12], distributed knowledge representation based on assembly neural networks [13-15] among others can be used to solve the automation problems. To introduce these methods into the industry it is necessary to examine them first in real industry environment. Such examination is very expensive. It is possible to reduce the examination cost by using cheaper environment that simulates all the processes of mechanical manufacturing. Such environment can be created on the basis of the micromechanical equipment. We have been developing micromechanical equipment for many years [16-18] and since this idea was proposed for the first time in [19] we have developed adaptive systems based on this idea and performed some experiments that prove the efficiency of the proposed approach. In this paper we describe the results that were obtained during last two years. Our adaptive systems for micromechanical equipment control are based on image recognition with neural networks.

This paper is organized in the following way: the second section contains the brief description of micromechanical devices that have been developed and used in our work. In the third section we describe image recognition systems based on neural networks and their applications in micromechanical equipment automation. The fourth section contains description of a new problem in micromechanical manufacturing that can be solved by the methods based on neural networks.

\section{Development of micromechanical equipment}

The main idea of low cost micromechanical equipment manufacturing is the following: each new micro device should be manufactured by micromachine tools and micro assembly devices which have the overall sizes comparable with the overall sizes of work pieces to be manufactured. For example, if new microdevice contains the shaft of $0.2 \mathrm{~mm}$ diameter and of $0.8 \mathrm{~mm}$ length then this shaft is to be manufactured with the lathe with the overall sizes of $4 \mathrm{~mm} * 4 \mathrm{~mm} * 4 \mathrm{~mm}$. In most cases the lathe of such overall sizes will automatically have tolerances that coincide with the shaft specifications. The main errors of machine tools that originate from thermal expansions, vibrations, elastic deformations etc. are proportional to the overall sizes of the machine tool [20]. So if we manufacture the micro shaft with the lathe that has 25 times smaller overall sizes than the overall sizes of the conventional lathe then we can decrease the tolerances 25 times. The low end lathe of conventional overall sizes has tolerances of about $0.05 \mathrm{~mm}$, so our micro lathe should have tolerances of about $0.002 \mathrm{~mm}$. It is sufficient for most applications of said micro shaft. Low end conventional lathe has low cost. Our micro lathe should have even lower cost due to low consumption of materials, work area and energy.

To examine the possibility of production of micromachine tools that have low cost components we have developed 2 prototypes of micromachine tools with the cost of components of less than $\$ 100$. One of these prototypes is shown in Fig. 1 .

The experiments with these prototypes have proved that they permit manufacturing of micro work pieces similar to ones produced with expensive Japanese micro lathe. Some examples of micro work pieces manufactured by our micromachine tools are presented in Fig. 2 and Fig. 3. 


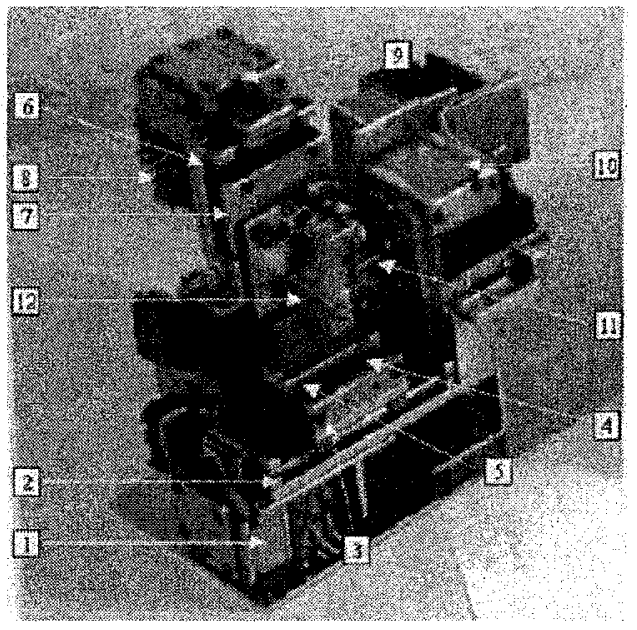

Fig. 1. Micromachine tool prototype: 1 - Y-direction drive; 2 - Y-direction guides; $3-X$ direction guides; 4 - X-direction carriage; 5 - X-direction drive; 6 - Z-direction guides; 7 - Zdirection carriage; 8 -Z-direction drive motor; 9 -Z-direction drive gearbox; 10 - chuck support; 11 - chuck; 12 - cutter and measurement tool support

Japanese researchers turned the brass needle of $0.05 \mathrm{~mm}$ diameter to show the possibility of micro turning [21]. They used micro lathe which has expensive components (some thousands dollars). We repeated their results (Fig. 2) with micromachine tool that has very cheap components (less than $\$ 100$ ).

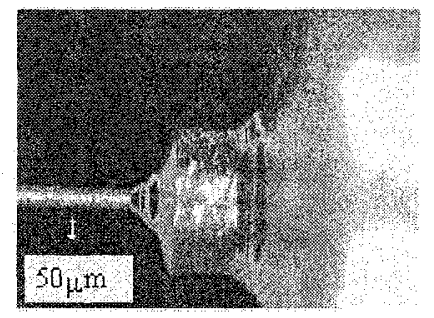

Fig. 2. Brass needle of $50 \mu \mathrm{m}$ diameter manufactured with the first micromachine tool prototype

In Fig. 3 two examples of micro work pieces manufactured with our micromachine tool prototype are presented. The first work piece is the gear with the worm that can be used in micro transmissions. The second work piece is the screw that can be used in gas filters of "micto cyclone" type. 

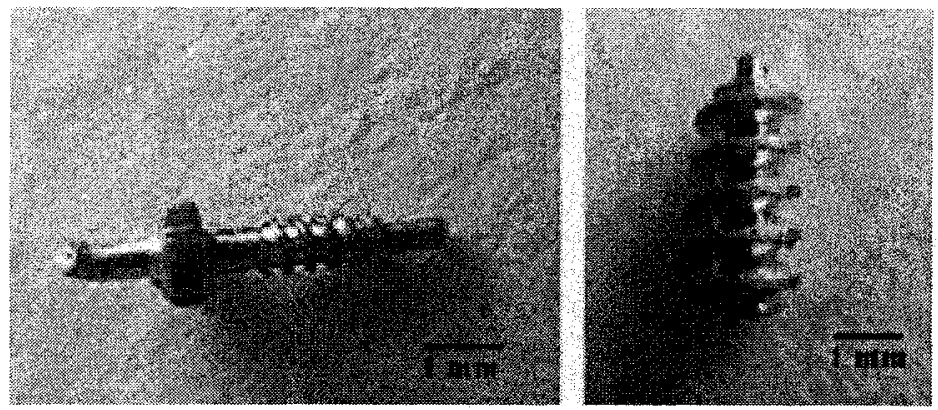

Fig. 3. Examples of micro work pieces manufactured with the first micromachine tool prototype

\section{Computer vision systems for micromechanical applications}

To obtain high precision in low cost microequipment we use adaptive algorithms based on computer vision systems.

\subsection{Image recognition in micro assembly}

The first problem which we dealt with was the problem of micro assembly. To introduce the pin into the hole it is necessary to collocate them with close tolerances. Low cost microequipment does not permit such collocation without adaptive algorithms. Neural network image recognition system was created to recognize the mutual pin-hole position. To investigate the potential of this system we created the image database that contains 441 images with different mutual positions of pin and hole. For this purpose we used the prototype shown in Fig. 4.

In our system we used one TV camera for teleconferences and four light sources [11]. The shadows from different light sources (Fig. 5) permit us to obtain the 3-D position of the pin relative to the hole. We use a neural classifier LIRA (Limited Receptive Area) to recognize this position [12]. The input of our neural classifier is an image that is a combination of four images of one pin-hole position that correspond to different light sources. This image is processed and the contours are extracted (Fig. 6). The output of the neural classifier gives the $X$ and $Y$ coordinates of pin relative to the hole.

If this position is recognized with the precision of $0.1 \mathrm{~mm}$ then the recognition rate is sufficiently high: $99.5 \%$ for $X$ coordinate and $89.9 \%$ for $Y$ coordinate.

\subsection{Shape recognition}




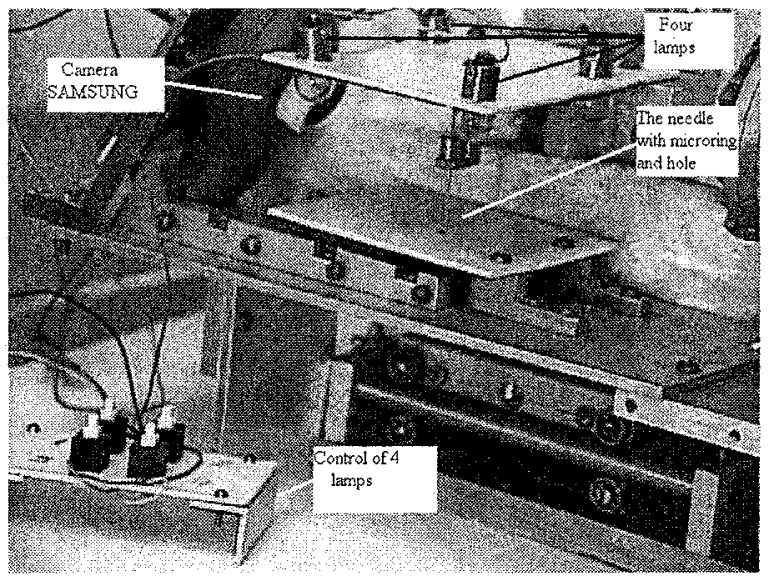

Fig. 4. Micro assembly system controlled by the vision system
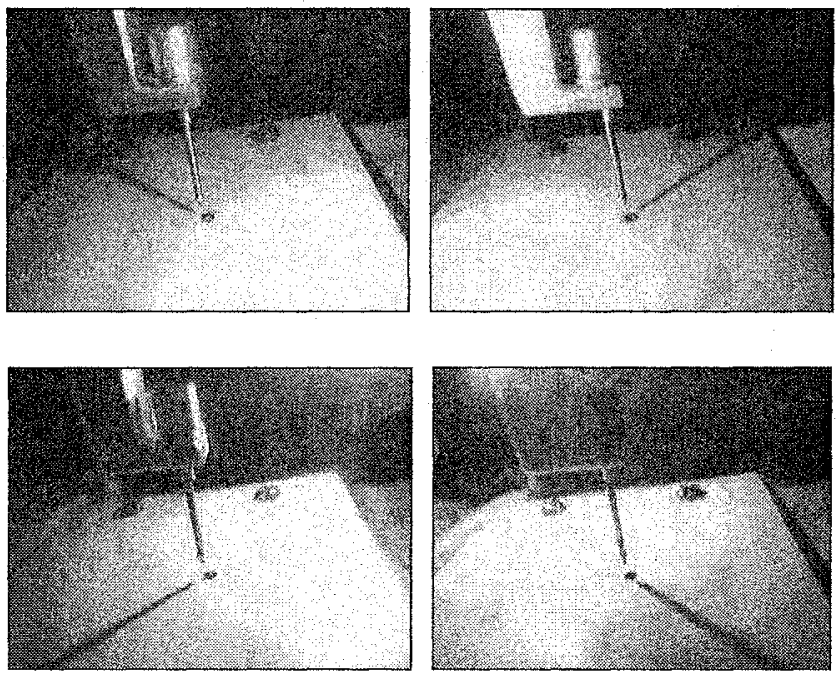

Fig. 5. An example of four images of the same pin-hole position obtained with different light sources

The other task where we used neural networks was the task of shape recognition [22]. Low cost microequipment does not permit precise allocation of the cutters in $\mathrm{CNC}$ lathe. The errors of cutter allocation produce erroneous shape of manufactured work piece. An example of such erroneous shape is shown in Fig. 7. Two cutters are used for manufacturing of the screws shown in Fig. 7. One cutter is used for the 
treatment of the outer diameter and the other one is used for the treatment of the thread. If mutual position of cutters is not correct the thread may have erroneous shape (Fig. 7, $a, c, d$ ).

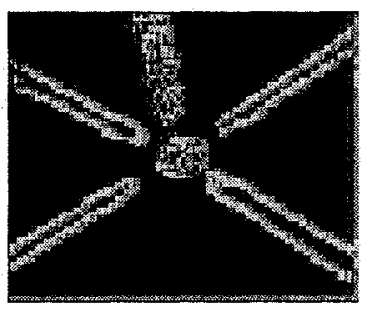

Fig. 6. An example of the input image of the LIRA neural classifier

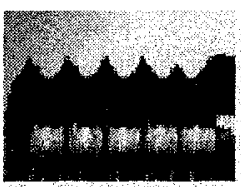

a

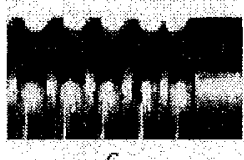

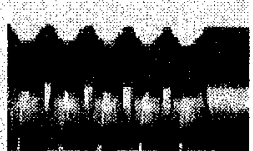

$b$

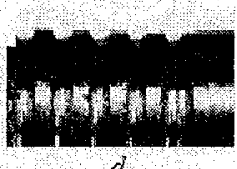

Fig. 7. Examples of micro screws manufactured with different mutual positions of cutters

It is difficult to evaluate the mutual position of cutters directly. That is why we propose to evaluate the correctness of their mutual position using the shape of the first screw that is manufactured with the lathe. If the distance between the second cutter and the lathe axis is less than necessary then the shape of the thread will be like the one presented in Fig. $7, a$. If this distance is larger than necessary then the shape of the thread will be like ones in Fig. 7, $c, d$.

We have produced 40 screws of $3 \mathrm{~mm}$ diameter with the $\mathrm{CNC}$-lathe Boxford. Ten screws were produced with correct position of the thread cutter (Fig. 15, b). Thirty screws were produced with erroneous positions of the cutter.

Ten of them (Fig. 15, a) had the distance between the cutter and the screw axis $0.1 \mathrm{~mm}$ smaller than necessary. Ten screws (Fig. 15, c) were produced with the distance $0.1 \mathrm{~mm}$ larger than necessary and the remaining ten (Fig. 15, d) with the distance $0.2 \mathrm{~mm}$ larger than necessary. We created an image database of these screws using web camera Samsung mounted on an optical microscope. Five randomly 
selected images from each group were used for the neural classifier's training and the other five were used for the neural classifier's testing.

The best recognition rate in shape recognition obtained with the $\mathrm{PCNC}$ neural classifier (Permutation Coding Neural Classifier) was 92.5\% [22].

\subsection{Texture recognition}

The third task was the recognition of surface textures. This is the issue of the day for the quality inspection systems and sometimes for work piece orientation recognition in the assembly process. For this task we developed the texture recognition system based on the RSC (Random Subspace) neural classifier [23].

To test our texture recognition system we created our own image database of metal surface images. Four texture classes correspond to metal surfaces after: milling, polishing with sandpaper and turning with lathe (Fig. 8).
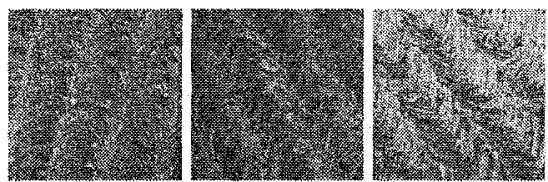

$a$
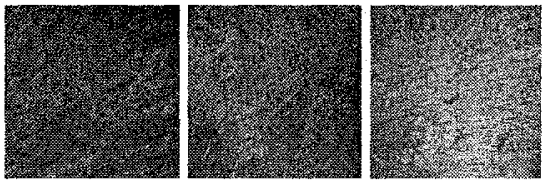

$b$
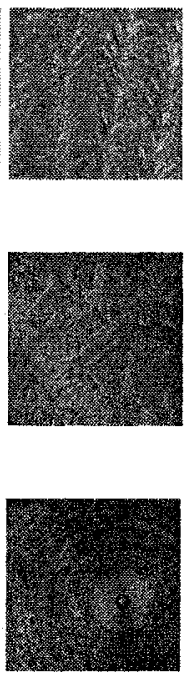

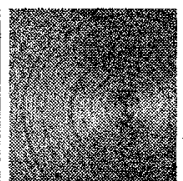

Fig. 8. Examples of metal surfaces after: $a$ ) milling, $b$ ) polishing with sandpaper, $c$ ) turning with lathe

It can be seen that different lighting conditions affect greatly the grayscale properties of the images. The textures may also be arbitrarily oriented and not centered perfectly. Metal surfaces may have minor defects and dust on it. All this image properties correspond to the conditions of the real industrial environment and make the texture recognition task more complicated. Scanning square window was used to extract texture features for neural classifier's training and testing. The numbers of images in training and recognition sets were 3 and 17 correspondingly. The best recognition rate of $80 \%$ was obtained in this task. 


\section{Future work: Avoiding the resonance oscillations in cutting process}

Mechanical treatment of metal surfaces frequently suffers from resonant oscillations of either work piece or cutting tool. Such oscillations result in decrease of both surface quality and work piece precision. The resonant oscillations appear when the rigidity of the system Machine tool - Tool - Work piece is insufficient. In Fig. 9, a an example of the work piece that was manufactured without resonant oscillation is presented.

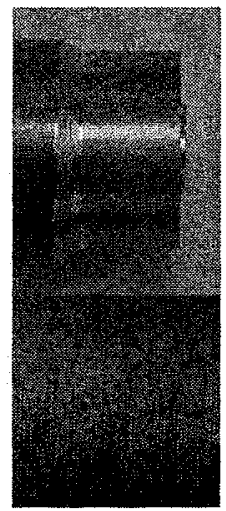

a

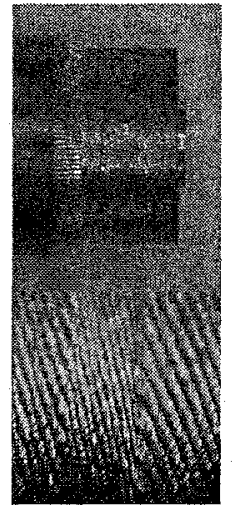

$b$

Fig. 9. Work piece and its surface: $a$ ) without resonant oscillations, b) with resonant oscillations

For comparison, an example of the work piece that was manufactured with resonant oscillation is presented in Fig. 9, b. Many factors affect the resonance oscillations appearance. We intent to prove the connectionist neural network approach for prediction of resonant oscillations. We also want to try to apply the adaptive critic design methods to the optimal control of cutting process under resonant oscillations condition [24].

\section{Acknowledgements}

This work was supported by projects PAPIIT IN108606-3, PAPIIT IN116306-3. 


\section{References}

1. Eberhart, R.: Overview of computational intelligence [and biomedical engineering applications]. Proceedings od the 20-th Annual International Conference of the IEEE Engineering in Medicine and Biology Society 3 (1998) 1125-1129

2. Hui, T., Brown, D., Haynes, B., Xinxian Wang: Embedded e-diagnostic for distributed industrial machinery. IEEE International Symposium on Computational Intelligence for Measurement Systems and Applications (2003) 156-161

3. Awadallah, M., Morcos, M.: Application of AI tools in fault diagnosis of electrical machines and drives-an overview. IEEE T Energy Conver 18, Ișsue 2 (2003) 245-251

4. Werbos, P.: Advanced Forecasting Methods for Global Crisis Warning and Models of Intelligence. In: General Systems Yearbook 22 (1977) $25-38$

5. Prokhorov, D., Wunsch, D.: Adaptive Critic Designs. IEEE T Neural Networ 8, N 5 (1997) 997-1007

6. Xiaoou Li, Lara-Rosano, F.: A weighted fuzzy Petri net model for knowledge learning and reasoning. International Joint Conference on Neural Networks, IJCNN $9942368-2372$

7. Xiaoou Li, Wen Yu, Lara-Rosano, F.: Dynamic Knowledge Inference and Learning under Adaptive Fuzzy Petri Net Framework. IEEE T Syst Man Cy 39, N4 (2000) 442-450

8. Bottou, L., Cortes, C., Denker, J., Drucker, H., Guyon L., Jackel L., LeCun J., Muller U., Sackinger E., Simard P., Vapnik V.: Comparison of Classifier Methods: a Case Study in Handwritten Digit Recognition. In: Proceedings of $12^{\text {th }}$ IAPR International Conference on Pattern Recognition 2 (1994) 77-82

9. Fukushima, K. Neocognitron: A hierarchical neural network capable of visulal pattern recognition. Neural Networks 1 (1988) 119-130

10. Roska, T., Rodriguez-Vazquez, A.: Toward visual microprocessors. Proceedings of the IEEE 90, Issue 7 (July 2002) 1244-1257

11. Baidyk, T.: Application of Flat Image Recognition Technique for Automation of Micro Device Production. Proceedings of the International Conference on Advanced Intelligent Mechatronics "AIM'01", Italy (2001) $488-494$

12. Baidyk, T., Kussul, E., Makeyev, O., Caballero, A., Ruiz, L., Carrera, G., Velasco, G.: Flat image recognition in the process of microdevice assembly. Pattern Recogn Lett 25/1 (2004) $107-118$

13. Kussul E.M., 1992, Associative Neural Structures. Kiev, Naukova Dumka, 144p. (in Russian).

14. Amosov N.M., Baidyk T.N., Goltsev A.D., Kasatkin A.M., Kasatkina L.M., Kussul E.M., Rachkovski D.A., 1991, Neurocomputers and Intelligent Robots. Kiev, Naukova Dumka, 272 p. (in Russian).

15. Baidyk T.N., 2001, Neural Networks and Artificial Intelligence Problems. Kiev, Naukova Dumka, 264 p. (in Russian).

16. Kussu1, E., Rachkovskij, D., Baidyk, T., Talayev, S.: Micromechanical Engineering: a Basis for the Low-cost Manufacturing of Mechanical Microdevices Using Microequipment. J Micromech Microeng 6 (1996) 410-425

17. Kussul, E., Baidyk, T., Ruiz-Huerta, L., Caballero, A., Velasco, G., Kasatkina, L.: Development of Micromachine Tool Prototypes for Microfactories. J Micromech Microeng 12 (2002) 795-812.

18. Kussul E., Baidyk T., Ruiz L., Caballero A., Velasco G., 2002, Development of Low-cost Microequipment. 2002 International Symposium on Micromechatronics and Human Science, IEEE, Nagoya, Japan, Oct. 20-23, pp.125-134.

19. Lara-Rosano F., Kussul E., Baidyk T., Ruiz L., Caballero A., Velasco G., 2004, Artificial Intelligence Systems in Micromechanics. In: Artificial Intelligence Applications and Innovations (IFIP $18^{\text {th }}$ Computer Congress), Ed. By Max Bramer, Vladan Devedzic Boston/Dordrecht/London, Kluwer Academic Publishers, pp.1-10 
20. Kussul E., Baidyk T., Ruiz-Huerta L., Caballero-Ruiz A., Velasco G., 2006, Scaling down of microequipment parameters, Precis Eng (to be published).

21. Yuichi Okazaki, and Tokio Kitahara, Micro-lathe equipped with closed-loop numerical control, $2^{\text {nd }}$ International Workshop on Microfactories IWMF2000, Fribourg, Switzerland, October $9-10,2000, \mathrm{pp} .87-91$

22. Baidyk T., Kussul E., 2004, Neural Network Based Vision System for Micro Workpieces Manufacturing. WSEAS T Syst, Issue 2, Volume 3, April 2004, pp.483-488.

23. Baidyk T., Kussul E., Makeyev O. Texture Recognition with Random Subspace Neural Classifier, WSEAS T Circuits Syst, Issue 4, Volume 4, April 2005, pp.319-325.

24. Werbos P. Approximate Dynamic Programming for Real-Time Control and Neural Modeling. Chapter 13 in Handbook of Intelligent Control: Neural, Fuzzy and Adaptive Approaches, (White \& Sofge, eds.), Van Nostrand Reinhold, New York, NY, 1992. 